\title{
The risk analysis of reservoir operation based on uncertain theory
}

\author{
Ke-fei $\mathrm{LI}^{1,}$, , Zheng-mao Jia ${ }^{1, a}$, Li-yuan $\mathrm{He}^{1, \text { a }}$, Bao-jian Hou ${ }^{1, \text { a }}$ \\ ${ }^{1}$ Yellow River Engineering Consulting Co. , Ltd , Zhengzhou 450003 , P. R. China \\ a984547253@qq.com
}

\begin{abstract}
Keywords: uncertain theory; chance-constrained; genetic algorithm (GA); hybrid intelligent algorithm; the Three Gorges

Abstract. Uncertain theory is a new tool to quantitatively study the risk analysis under the uncertain condition. Based on uncertain theory and combined with actual characteristics of reservoir operation, the risk analysis model of reservoir operation was established and a hybrid intelligent algorithm coupling uncertainty simulation technology and genetic algorithm was designed. Through the application of the Three Gorges, the results showed that the model can provide some technical support for the dispatchers to optimize the decision-making considering the risk situations.
\end{abstract}

\section{Introduction}

Reservoir operation process which widely exists a series of uncertainties such as randomness, fuzziness, unascertained, gray and so on, is a very complex multi-dimensional nonlinear system [1,2]. Where randomness is a kind of objective uncertainty and often uses probability risk analysis and stochastic simulation method to solve the risk problems of stochastic system [3]; Fuzziness is a kind of subjective uncertainty and often uses fuzzy risk analysis methods to study the risk of fuzzy system[4]; For the uncertainties in the random and fuzzy environment, often uses random fuzzy theory to study the risk in the system. But for the general uncertain systems which are neither purely random systems nor purely fuzzy systems should not be used probabilistic risk or fuzzy risk analysis methods. Uncertainty theory is a new mathematical axiomatic system to deal with the uncertain phenomena and also is a new tool to quantitatively study the risk analysis under the uncertain condition[5,6]. This theoretical system provides the necessary theoretical and methodological foundation for dealing the risk analysis problems under the uncertain conditions.

\section{The risk analysis model of reservoir operation based on uncertain theory}

\section{Uncertain theory}

Set the $\Gamma$ is a collection of non empty and $L$ is a $\sigma$ algebra which is composed of some subsets in the $\Gamma$, called the elements in the $L$ as event. For each event $\Lambda$, definite a non negative number $M\{\Lambda\}$, and there are four axioms as follows.

Axiom 1: $\mathrm{M}$ is according to normative, that is: $\mathrm{M}\{\Gamma\}=1$;

Axiom 2: $M$ is monotonically increasing, that is: when $A \subset B$, there is $M\{A\} \leq M\{B\}$;

Axiom 3: $\mathrm{M}$ is according to the self duality, that is: for each event $\Lambda$, there is $\mathrm{M}\{\Lambda\}+\mathrm{M}\left\{\Lambda^{c}\right\}=1$;

Axiom 4: $\mathrm{M}$ is according to Second countable additive, that is: for $\forall\left\{\Lambda_{i}\right\}_{i=1}^{\infty} \subset L$, there is $M\left\{\bigcup_{i=1}^{\infty} \Lambda_{i}\right\} \leq \sum_{i=1}^{\infty} M\left\{\Lambda_{i}\right\}$

If the set function $M\{\Lambda\}$ accords to these four axioms, call $M$ uncertain measure, at the same time call the triples $(\Gamma, L, \mathrm{M})$ uncertain space.

Set that there are $\mathrm{n}$ uncertain space $\left(\Gamma_{k}, L_{k}, \mathrm{M}_{k}\right)(k=1,2, \ldots, n)$, and $\Gamma=\Gamma_{1} \times \Gamma_{2} \times \ldots \times \Gamma_{n}, L=L_{1} \times L_{2}$ $\times \ldots \times L_{n}$.

Axiom 5: set that the $\mathrm{M}_{k}$ is a uncertain measure on the non empty set $\Gamma_{k}$, so the product measure of $\Gamma$ is: 


$$
\mathrm{M}\{\Lambda\}=\left\{\begin{array}{lll}
\sup _{\Lambda_{1} \times \Lambda_{2} \times \cdots \times \Lambda_{n} \subset \Lambda^{1}} \min _{1 \leq k \leq n} \mathrm{M}_{k}\left\{\Lambda_{k}\right\}, & \text { if } & \sup _{\Lambda_{1} \times \Lambda_{2} \times \cdots \times \Lambda_{n} \subset \Lambda} \min _{1 \leq k \leq n} \mathrm{M}_{k}\left\{\Lambda_{k}\right\}>0.5 \\
1-\sup _{\Lambda_{1} \times \Lambda_{2} \times \cdots \times \Lambda_{n} \subset \Lambda^{c}} \min _{1 \leq k \leq n} \mathrm{M}_{k}\left\{\Lambda_{k}\right\}, & \text { if } & \sup _{\Lambda_{1} \times \Lambda_{2} \times \cdots \times \Lambda_{n} \subset \Lambda^{c}} \min _{1 \leq k \leq n} \mathrm{M}_{k}\left\{\Lambda_{k}\right\}>0.5 \\
0.5, \quad \text { others } & &
\end{array}\right.
$$

Where: $\Lambda \in \mathbf{L}, \mathbf{M}=\mathrm{M}_{1} \wedge \mathrm{M}_{2} \wedge \ldots \wedge \mathrm{M}_{n}$..

Uncertain variables are real-valued measurable function in the uncertain measure space, it is an effective mathematical tool to characterize and describe the uncertain phenomena. In describing and measuring the risk, because of the risk of it has a certain degree of uncertainty, we often use the uncertain variables to describe the risk under the uncertain environment and at the same time said the uncertain variables which characterized the risk as risk variables. From the perspective of mathematical functions, there is no uniform order relation between the risk variables and the mutual size cannot be directly compared, therefore it can only use the expected value, measure value, critical value, etc digital features of the risk variables to compare and quantify in certain mathematical sense. And following, based on uncertain theory and combined with actual characteristics of reservoir operation, the risk analysis model of reservoir operation will be established.

\section{The risk analysis model of reservoir operation}

Based on the uncertain theory, from the perspective of chance risk value, considering of the power requirements of the hydropower station reservoir, because the process of generation scheduling may be subjected to numerous uncertainties, that may cause the power station cannot meet the objective requirements thus lead to risk loss. This paper mainly consider the generation impact of the hydropower station reservoir caused by the uncertainty of the runoff, and let the runoff be risk variable, allowing the power requirement violates the constraint conditions to a certain extent, but the uncertainty measure of constraint violations cannot exceed the given certain confidence level, i.e. the measure (risk) of violation constraints is not more than 1-confidence level. Scheduling period in years, calculation time in days, let the generating capacity of the reservoir be maximum in the scheduling period as the goal, the chance risk value model of the Hydropower generation scheduling was established.

Objective function:

$$
\begin{gathered}
\max \bar{f} \\
f=\sum_{t=1}^{T} N_{t} \Delta t=\sum_{t=1}^{T} K q_{t} H_{t} \Delta t
\end{gathered}
$$

Where: $N_{t}$ is the output in period of time $t$ of the hydropower station reservoir; $\Delta t$ is the time length; $T$ is the total number of periods; $\mathrm{K}$ is the coefficient of output; $q_{t}$ is the power flow in period of time $\mathrm{t}$, $H_{t}$ is the power average head in period of time $\mathrm{t}$.

Constraint conditions:

(1) Constraint of the objective function

$$
M\{f \geq \bar{f}\} \geq \alpha
$$

Where: $\alpha$ is the given certain confidence level, $\mathrm{M}\{\ldots\}$ represents the variable measure of the event $\{\ldots\}$. The probability of the constraint condition of objective function is the risk of the generation dispatch. The decision-makers of the reservoir operation management should choose a reasonable objective constraint confidence level in the range of $[0,1]$. The lower of the confidence level, the greater risk of the decision-maker faces. Others, the higher of the confidence level, the smaller risk of the decision-maker faces.

(2) Chance constraint of the reservoir storage

$$
M\left\{V^{\min } \leq V_{t} \leq V^{\max }\right\} \geq \beta
$$

Where: Considering the impact of runoff uncertainty on the reservoir storage capacity, the constraint of the reservoir storage capacity will be viewed as chance constrained. $V_{t}$ is the storage 
capacity in period of time $t, V^{\min }$ is the dead storage capacity of the reservoir, $V^{\max }$ is the storage capacity corresponding to the normal water level and corresponding to the flood control level in flood season. $\beta$ is the given certain confidence level of the reservoir storage capacity constraint.

(3) Water balance constraint

$$
V_{t+1}=V_{t}+\left(q_{t}-Q_{t d}\right) \Delta t
$$

Where: $q_{t}$ is the average inflow of the reservoir in period of time $t, V_{t}, V_{t+1}$ are respectively the reservoir storage capacity in period of time $t, t+1 ; Q_{t d}$ is the average discharge of the reservoir in period of time $t$.

(4) The discharge constraint

$$
Q_{t}^{\min }<Q_{t d}<Q_{t}^{\max }
$$

Where: $Q_{t d}$ is the average discharge of the reservoir in period of time $t ; Q_{t}^{\min }, Q_{t}^{\max }$ are respectively the minimum, maximum discharge of the reservoir allowed.

(5) Non-negative constraints, etc.

\section{Solution of the model}

\section{Uncertainty simulation technology}

In the above risk analysis model established based on uncertain theory, there are a lot of uncertain constraints. When deal with the uncertain constraints, the uncertain simulation technology is very effective. Take the uncertainty measure constraint $\mathrm{M}\left\{g_{j}(x, \xi) \leq 0, j=1,2, \ldots, n\right\} \geq \beta$ as an example to introduce the processing method of uncertain simulation technology. Where: $x$ is the decision vector; $\xi$ is the uncertain risk variable. The specific steps of the uncertain simulation technology testing constraints are as follows:

1) Generate $N$ independent random vectors $\left(\xi_{1}, \xi_{2}, \ldots, \xi_{N}\right)$ from the sample space of the uncertain variable $\xi$

2) Hypothesize that $N^{\prime}$ is the number of meeting the constraint in the $N$ independent random vectors and set $N=0$;

3) If $g_{j}\left(x, \xi_{i}\right) \leq 0,(1 \leq i \leq N)$, then $N^{\prime}=N^{\prime}+1$;

4) By the law of large numbers, using frequency $N^{\prime} / N$ as the estimation of the uncertainty measure. If $N^{\prime} / N \geq \beta$, then seemed to satisfy the constraints; else didn't seem to satisfy the constraints and end the testing.

\section{The designing of the algorithm}

For the uncertain risk analysis model, using the traditional algorithm to solve is difficult, so coupled uncertainty simulation technology and genetic algorithm, a hybrid intelligent algorithm was designed to solve the uncertain risk analysis model. The basic solving steps of the hybrid intelligent algorithm are as follows:

1) Generate the input and output data for the uncertain function in the model using the uncertain simulation technology;

2) Input parameters used in the model, for example population size, crossover probability, mutation probability and the number of iterations, etc.

3) Initialize pop-size chromosomes and use uncertainty simulation technology to test the feasibility of the chromosomes, and then generate $N$ feasible chromosomes meeting the constraint;

4) Update the chromosome through crossover and mutation operations and use uncertainty simulation technology to test the feasibility of the progeny chromosomes;

5) Calculate the target value of all the chromosomes;

6) Calculate the fitness of each chromosome by the target value;

7) Select the chromosomes by rotating the roulette wheel; 
8) Repeat steps 4) to step 7) until completing the given number of cycles and give the best chromosome as the optimal solution of the problem.

The basic process of the hybrid intelligent algorithm designed is shown in figure 1.

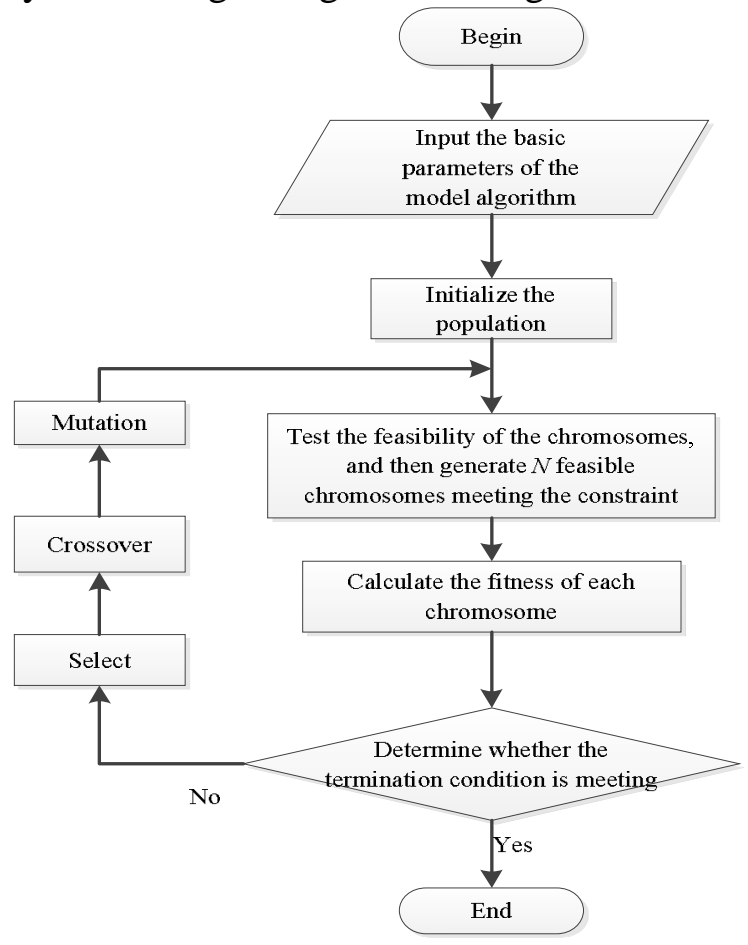

Fig.1 Basic calculation process of the hybrid intelligent algorithm

\section{Application of the example}

The Three Gorges reservoir is a large reservoir in the upper reaches of the Yangtze River, which the dead water level is $145 \mathrm{~m}$, the normal water level is $175 \mathrm{~m}$, the flood water level is $145 \mathrm{~m}$, and the total storage capacity is 393 billion $\mathrm{m} 3$, the regulating capacity is 165 billion $\mathrm{m} 3$, the flood control capacity is 221.5 billion $\mathrm{m} 3$. When solve the chance risk value model, stochastic simulate the inflow of the Three Gorges reservoir by the normal distribution considering adjacent time effect[7,8]. Parameters in hybrid intelligent algorithm are set as follows: the population size is 50 , the crossover probability is 0.8 , mutation probability is 0.025 , and the maximum number of iterations is 1500 . To discuss the basic characteristics of the model, calculate the scheduling scheme in the different risk level (confidence level), and then get the effect of the different risk on scheduling scheme. The calculation results are shown in Table 1.

Table.1 Effect of different risk on scheduling schemes

\begin{tabular}{|c|c|c|c|}
\hline \multirow[b]{2}{*}{ Schemes } & \multicolumn{2}{|c|}{ Confidence levels } & \multirow{2}{*}{$\begin{array}{l}\text { Power generation target profit } \\
\text { value }\left(10^{8} \mathrm{~kW} \cdot \mathrm{h}\right)\end{array}$} \\
\hline & Constraint of object function $\alpha$ & Constraint of storage capacity $\beta$ & \\
\hline 1 & 0.80 & 0.90 & 891.68 \\
\hline 2 & 0.75 & 0.90 & 897.54 \\
\hline 3 & 0.70 & 0.90 & 911.18 \\
\hline 4 & 0.65 & 0.85 & 921.95 \\
\hline 5 & 0.60 & 0.80 & 930.09 \\
\hline 6 & 0.60 & 0.75 & 933.68 \\
\hline 7 & 0.60 & 0.70 & 934.85 \\
\hline
\end{tabular}

As can be seen from Table 1, under the different confidence levels, the possible target profit value of the reservoir dispatching system achieved is different. Analyzing the scheme of 1 to 3 , when fix the level of reservoir storage capacity constraint $\beta=0.90$, along with the constraint level of the power object function is gradually reducing; the power generation target profit value of the reservoir achieved is gradually increasing. The higher of the confidence constraint level is, the smaller risk of the reservoir system faces, so the increase of the power generation target value is based on the risk increase of the 
reservoir system faces. Analyzing the scheme of 5 to 7, when fix the level of power object function constraint $\alpha=0.60$, along with the constraint level of reservoir storage capacity is gradually reducing; the target profit value of the reservoir achieved is gradually increasing. The constraint level of reservoir storage capacity reflects the flood risk of the reservoir and the smaller of the constraint level, the higher of the flood risk of the reservoir system faces, so the increase of the power generation target value is based on the increase of the flood risk, and this shows that there is a contradiction between the flood control and power generation of the Three Gorges reservoir.

The chance risk value model can help the decision-maker determine a reasonable target generating value according to the different risk situations, and then the management of uncertain risk problem in the process of reservoir generating operation can be realized. Take the scheme of 4 as an example, when the given confidence levels of the objection function constraint and reservoir storage capacity constraint are respectively 0.65 and 0.85 , as can be seen from Table 1 , considering the effect of the runoff random, now the power generation target profit value set at 92.195 billion $\mathrm{kW} \cdot \mathrm{h}$ is more reasonable. Therefore, the management of reservoir operation can choose appropriate confidence constraint level according to the reservoir facing uncertainty factors, and select the hydropower generation scheduling scheme to guide the reservoir scheduling.

\section{Conclusions}

(1) Based on uncertain theory and combined with actual characteristics of reservoir operation, the chance risk value model of reservoir operation was established;

(2) In view of the large number of uncertain measure constraints in the model, a hybrid intelligent algorithm coupling uncertainty simulation technology and genetic algorithm was designed;

(3) Through the application of the Three Gorges, the model can prior compare the benefits and risks of the cascade hydropower stations and further enrich and improve the risk management theoretical system of the reservoir operation.

\section{Acknowledgements}

This work was financially supported by the National Key research and development program of China (No. 20170404406).

\section{References}

[1] Li-ping WANG, Yan-ke ZHANG, Chang-ming JI, etc. Risk calculation method for complex engineering system[J]. Water Science and Engineering, 2011, 4(3): 345-355

[2] LIU Pan, ZHANG Wenxuan, LI Tianyuan. Derivations of risk-based reservoir operation rule curves[J]. Journal of Hydroelectric Engineering, 2013, 32(4): 252-259

[3] WANG Liping, ZHANG Yanke, JI Changming, etc. Simulate maximum entropy method and its application to reservoir flood risk calculation [J]. Journal of Hydraulic Engineering, 2011, 42(1): 27-32

[4] LI Kefei, JI Changming, ZHANG Yanke, etc. Fuzzy Risk Analysis of Reservoir Predicted Power Generation Operation [J]. Water Resources and Power, 2012, 30(12): 44-47

[5] Liu B., Uncertainty Theory [M]. 2nd ed., Springer-Verlag, Berlin, 2007

[6] LIU Baoding, PENG Jin. Uncertainty Theory Tutorial[M]. Beijing: Tsinghua University press, 2005

[7] JI Changming, LI Kefei, ZHANG Yanke, etc. Stochastic multi-objective decision-making model of the reservoir operation based on chance-constrained programming[J]. Power System Protection and Control, 2012, 40(19): 36-40 
[8] ZAMBELLI M, SIQUEIRA T G, CICOGNA M, et al. Deterministic versus stochastic models for long term hydrothermal scheduling// Proceedings of 2006 IEEE Power Engineering Society General Meeting, Jun 18-22, 2006, Montreal, Canada 